\title{
Tangence
}

\section{Le religieux et le fondement du politique}

\section{Jacques Pierre}

Numéro 63, juin 2000

Fictions et politique

URI : https://id.erudit.org/iderudit/008180ar

DOI : https://doi.org/10.7202/008180ar

Aller au sommaire du numéro

Éditeur(s)

Presses de l'Université du Québec

ISSN

0226-9554 (imprimé)

1710-0305 (numérique)

Découvrir la revue

Citer cet article

Pierre, J. (2000). Le religieux et le fondement du politique. Tangence, (63), 7-27.

https://doi.org/10.7202/008180ar d'utilisation que vous pouvez consulter en ligne.

https://apropos.erudit.org/fr/usagers/politique-dutilisation/ 


\section{Le religieux et le fondement du politique Jacques Pierre, Université du Québec à Montréal}

\section{Introduction}

L'idée que les sphères du religieux et du politique puissent conjoncturellement rejaillir l'une sur l'autre n'est décidément pas nouvelle et a donné lieu, de la part des exégètes de l'histoire événementielle, à une littérature dont l'abondance se passe de tout commentaire. Mais le rapport entre le religieux et le politique ici étant incident, certains ont pu croire, à la faveur d'une modernité où le religieux devait inéluctablement retraiter hors de la sphère publique avant de s'évanouir définitivement, que ce dernier pourrait être relégué aux archives de l'histoire politique en perdant son actualité, et s'y faire oublier ${ }^{1}$. La débat rationnel devait tôt ou tard supplanter complètement l'attestation de la croyance comme mode et principe d'agrégation du corps social. Sur la pente des choses et de l'histoire, l'opérativité rationnelle devait succéder aux incantations de la foi aussi infailliblement que le boulet lâché sur une déclivité la dévale. La disparition du religieux était simplement programmée dans l'ordre des choses.

Mais on sait qu'il en fut autrement; et depuis un certain temps déjà, l'actualité, se charge tous les jours de démentir nos augures. Non seulement le religieux n'est pas disparu de l'espace public mais sa montée ne s'est pas soldée pas par un reflux de la rationalité. En sorte que l'on a vu de biens étranges amalgames se constituer au fil des circonstances sociales et politiques: les technologies prospérer au sein des intégrismes les plus anachroniques, le langage scientifique s'associer à la gnose dans des institutions qu'on aurait pu croire immunisées contre de telles greffes, les sciences administratives user de la spiritualité comme d'une technique de gestion du personnel susceptible d'augmenter le rendement.

Et puis, ailleurs encore, dans de nombreuses régions du monde, le religieux s'est offert pour être le vecteur de la protestation sociale: en Pologne où l'Église catholique fut non seulement

1. Danielle Hervieux-Léger, La religion pour mémoire, Paris, Cerf, 1993. Tout particulièrement le chapitre intitulé "La sociologie contre la religion?". 
8

la seule force politique organisée contre le pouvoir communiste mais aussi un bastion identitaire capable d'assurer, en dépit de l'occupation soviétique, un trait d'union avec le passé glorieux de la nation; en Amérique du Sud où la théologie de la libération a fourni au projet égalitaire du marxisme la ferveur et l'enracinement populaire pour lutter contre l'oppression économique et culturelle des élites locales; dans le monde arabe où l'intégrisme musulman est devenu, après l'échec retentissant du marxisme, l'ultime recours non seulement contre la domination économique de l'Occident mais aussi contre l'aliénation de la référence à soimême. Chaque fois la religion survient ici comme un puissant adjuvant identitaire, voire comme la matrice même où est fondu le collectif. Dépositaire de la mémoire commune, la religion fournit non seulement l'ensemble des représentations autour desquelles le groupe se rassemble, mais elle semble aussi constitutivement associée à la production des conditions de possibilité de l'être social aussi bien que politique.

D'aucuns attribueront cette recrudescence de la religion à une rechute momentanée du patient dans son processus de convalescence - après tout, notre époque nous a habitués à déconvenir d'autres prophéties idylliques dont la réalisation a été reportée à plus tard, voire carrément suspendue - et voudront croire que, la tectonique des grandes mouvances historiques étant plus fondamentale que les tics passagers de la conjoncture, les choses reprendront tôt ou tard leur cours. Pourtant, la résurgence ou la perdurance des grandes mouvances religieuses dans le social et le politique ne laisse pas d'être une énigme qui interroge fondamentalement la pensée politique.

Il faut se demander en effet si le politique n'est pas fondé sur des mécanismes où la religion a une part essentielle à jouer, ou plutôt si les mécanismes en jeu dans le fondement d'un ordre politique ne sont pas ceux-là mêmes à l'œuvre dans toutes religions. Dans lequel cas, ce ne serait plus les traditions religieuses ou la religion en tant que telle qui seraient interpellées par ce questionnement mais le religieux lui-même. Les religions instituées ne seraient qu'un cas de figure parmi d'autres de la façon dont le religieux fonde un ordre politique. Le religieux serait moins un contenu mythologique et rituel identifiable à une tradition croyante - encore que la chose ne soit pas exclue - que la production spontanée de mythes et de rituels idoines par lesquels passe nécessairement un ordre politique qui se fonde. Et alors, il 
s'agirait non pas d'une mythologie qui aurait été empruntée sur le marché des représentations religieuses disponibles, mais une mythologie sur mesure dont le politique aurait transposé spontanément les contenus - grand soir rouge, nouvel ordre mondial, main invisible du marché ${ }^{2}$ — en se conformant aux mêmes mécanismes fondationnels que les traditions religieuses.

À l'évidence, le contenu de cette mythologie serait différent de celui produit par les confessions traditionnelles; mais il ne le serait pas essentiellement; en tout cas, il ne le serait pas par sa forme. Le "culte de la personnalité " que cultivent par exemple les dictatures de droite comme de gauche n'abolit pas la requête de transcendance à l'œuvre dans toute culture, mais il en fait simplement varier les modalités. Du reste, l'expression "culte de la personnalité" que l'on emploie pour désigner l'objet autour duquel sont souvent édifiées les dictatures ne procède que de la surdétermination de notre propre position politique. On dénonce les idoles adorées par les "sauvages" d'en face pour ne pas voir où sont érigés les autels de nos démocraties. À cet égard, le refus souvent obstiné de poser à nouveau la question de la place du religieux dans le social, l'acharnement à considérer cette question comme définitivement résolue, le soupçon de prosélytisme religieux — et le mépris qui l'accompagne — qui frappe immanquablement ceux qui la posent malgré tout, et enfin l'attente d'une confirmation à venir qui scellera définitivement le sort de la religion et achèvera de dénoncer son inanité, ont eux-mêmes quelque chose de vaguement religieux en rapport avec les conditions de possibilité du paradigme en vogue présentement dans les sciences humaines. Disons-le clairement: l'antagonisme entre les sciences humaines et la religion a souvent lui-même l'allure d'une guerre de religions.

Mais laissons cela à une autre fois. Notre propos ici est différent. Il veut interroger justement les conditions de possibilité du politique en général et l'affinité profonde du fait socio-politique avec le religieux.

2. Raoul Girardet, Mythes et mythologie politique, Paris, Seuil, 1986; Élyse Marienstras, Les mythes fondateurs de la nation américaine. Essai sur le discours idéologique aux États-Unis à l'époque de l'indépendance, 1763-1800, Paris, Maspero, 1976; Peter Ludwig Berger, The Sacred Canopy. Elements of a Sociological Theory of Religion, Garden City (New York), Doubleday, 1967, et Affrontés à la modernité. Réflexions sur la société, la politique, la religion, Paris, Éditions du Centurion, 1980. 


\section{L'instauration d'un ordre politique}

Qu'est-ce qui assure la fondation d'un ordre politique quelconque? Trois choses : d'abord un cadre normatif où sont déposées les représentations et où sont définies les règles du représentable; ensuite des moyens qui permettent d'inscrire ce cadre dans ce que, faute de mieux, nous appellerons pour l'instant, la réalité ; et enfin, un événement qui permet d'attester ce cadre normatif dans l'intersubjectivité.

En ce qui concerne le cadre normatif, on peut noter brièvement qu'il est la forme même de l'expérience où le donné sensible est organisé et articulé pour faire un monde ${ }^{3}$. Autant dire alors qu'il est coextensif à la culture dans son ensemble où il régit l'ensemble de la pensée et de l'action, et qu'il quadrille aussi bien notre rapport au monde matériel que celui à nous-mêmes et aux autres. Sous-ensemble de celui-ci, l'ordre politique concerne plus spécifiquement la forme de la vie collective, la conception que les uns et les autres se font du groupe, du bien commun, des rapports de dépendance qui irriguent le corps social et des formes d'autorité qui le hiérarchisent. Ce cadre normatif est donc l'objet d'une inscription dans le réel et d'une attestation dans la communauté. De l'inscription dépend l'effet de réalité; et de l'attestation dépend l'effet de vérité.

Or toute société tend à faire coïncider dans son propre fondement l'effet de vérité et l'effet de réalité ${ }^{4}$ en sorte que l'arbitraire historique et géographique de son existence peut être surmonté et érigé au rang de destin, d'élection et de prédestination. Et c'est là précisément, dans ce rabattement de la réalité sur la vérité, que les ressources de la mythologie et de la ritualisation sont

3. On aura compris que notre perspective est résolument constructiviste et s'inscrit dans la filiation de penseurs comme Martin Heidegger, Michel Foucault, Thomas Kuhn pour lesquels il n'y a pas d'une part la vérité originelle du réel et d'autre part son expression approximative et dérivée dans le langage, mais un seul monde où langage et réel sont respectivement "forme" et "substance" de l'expérience. Sur ce sujet, on se reportera à l'épistémologie de Louis Hjelmslev dans Prolégomènes à une théorie du langage [1943], Paris, Minuit, 1971.

4. Dans son anthropologie religieuse, Savoir local, savoir global [1983], Paris, PUF, 1986, Clifford Geertz différencie "modèle de" et "modèle pour". Le "modèle de" correspond à l'ensemble des représentations du monde et le "modèle pour" désigne les structures transformatrices du monde. Or la religion inscrit "modèle de" et "modèle pour" dans un rapport de circularité et de confirmation réciproque. 
mises en ouvre. La marque à partir de laquelle commence l'inscription dans le réel devient événement d'une attestation.

\subsection{L'inscription dans la réalité}

Dans le mesure où ce cadre normatif n'est pas un dispositif surajouté et second par rapport à l'expérience, il suppose, pour être vrai, les moyens de son incarnation dans le réel. Et si tant est qu'il n'y a pas de solution de continuité entre lui et le réel, les intimations du monde doivent pouvoir retentir et prendre place en lui; de même qu'il doit pouvoir réciproquement laisser son empreinte sur le monde. Le cadre normatif attend donc de son inscription l'effet de réalité sans lequel il dériverait sur le monde comme une fiction délirante.

Or, cela suppose des moyens de diverses natures: moyens heuristiques, technologiques, productifs et policiers; moyens qui peuvent être modestes ou considérables, être ceux d'une culture lithique ou d'une civilisation technologiquement avancée, mais qui impliquent tous l'exercice d'une certaine forme de coercition, subtile ou brutale, à l'égard du monde où, pour incarner justement ce cadre normatif, les corps sont dressés et le cours des choses infléchi ${ }^{5}$. Par le fait de l'inscription, l'Être est convoqué pour devenir substance, matière première, énergie et force de travail; la pensée est quadrillée par les formes du raisonnement, l'espace balisé par les géomètres, la nature domestiqué par les éleveurs, soumis à la question par les scientifiques ou par les inquisiteurs, pour que le monde devienne la réalisation de ce cadre normatif et en remplisse la forme disponible. Non pas que le monde soit indéfiniment malléable et docile à toutes les sollicitations du cadre normatif, mais là où il résiste, il n'est pas représentable et n'a pas de nom. La représentation le contourne sans pouvoir le dire et se contente de le montrer ${ }^{6}$.

Comprenons bien: le cadre normatif ne peut pas tout faire, mais il doit faire et quand il le fait, à proportion de ses moyens,

5. On se reportera ici aux deux ouvrages de Michel Foucault, Les mots et les choses. Une archéologie des sciences humaines, Paris, Gallimard, coll. "Bibliothèque des sciences humaines", 1966, et Surveiller et punir. Naissance de la prison, Paris, Gallimard, coll. "Bibliothèque des histoires", 1975.

6. Cette distinction entre dire et montrer vient de Ludwig Wittgenstein, Tractatus logico-philosophicus [1921], Paris, Gallimard, 1961. 
c'est toujours pour se réaliser. Un cadre normatif ne peut pas être en excès ou en reste par rapport à la réalité. Et à supposer qu'il le soit, comme dans le messianisme par exemple, ce n'est jamais qu'une avance consentie sur sa réalisation à venir. Ainsi, un cadre normatif est toujours proportionnel à ses moyens sous peine de perdre l'effet de réalité dont il tient son autorité. Si les moyens sont modestes, l'inscription se contentera, à la limite, de la marque avec laquelle elle commence. Mais le cadre normatif ne pourra être sans elle.

Fut-elle rudimentaire et démunie, une culture commence toujours avec la marque de l'assujettissement qu'elle inscrit sur les corps et sur le monde et à partir de laquelle le cadre normatif ensuite se déploie. Le cadre normatif n'est à vrai dire que la systématisation de cette marque qui, en se dépliant, gagne en extension et en profondeur. Ses moyens auront beau être rudimentaires, ses représentations se déployer dans des contrées imaginaires et improbables, si le cadre normatif a cet ancrage minimal dans la marque, on saura qu'il parle du monde, ou plus encore qu'il est le monde. De ce point de vue, le jeu d'osselets que le devin jette sur le sol pour lire le monde ou le simple coup de hache de silex dans le tronc d'un arbre pour démarquer le territoire, pour minimaux qu'ils soient, ne sont pas essentiellement différents des gigantesques ouvrages aplanissant, détournant ou enjambant les reliefs du monde. Ils proviennent d'un arbitraire assumé qui commence à inscrire l'ordre de la parole dans celui des choses; qui fait se rencontrer, ici dans la poussière ou l'écorce d'un arbre, là dans le flanc d'une montagne, les mots et les choses. C'est là dans cette marque que le cadre normatif intersecte la réalité et amarre le monde des représentations à celui de ses objets. Et l'être socialisé est celui qui porte cette marque - scarification, tatouage, signe distinctif et d'appartenance — qui lui a été imprimé par son groupe; de la même façon que le monde habité est celui qui a été humanisé par l'érection d'une borne. L'entrée en culture est signalée par cette marque aussi bien sur les individus que sur le monde. La marque transforme les individus en sujet, l'espace en lieu et le temps en récit ${ }^{7}$.

7. Paul Ricœur, Temps et récit, 3 volumes, Paris, Seuil, coll. "L'ordre philosophique ", 1983-1985. Plusieurs voies de réflexion mériteraient d'être explorées à partir de ce point. Sur le plan éthologique, le marquage du territoire par l'urine ou les excréments chez les mammifères supérieurs constitueraient 
Une fois inscrite, la marque peut alors pénétrer de plus en plus profondément et se développer extensivement sur le monde. En se donnant une syntaxe, la marque se systématise et devient écriture. Ce faisant, elle épouse de moins en moins le monde et le modèle de plus en plus. Le cadre normatif sera d'autant plus étendu et resserré autour du monde qu'il pourra aligner des moyens puissants susceptibles d'aplanir les résistances de la réalité. Domestication de la matière et des foules où la marque d'abord posée devient un coin que l'on enfonce dans la réalité. Une société sera d'autant plus sûre de son bon droit et de sa propre vérité que ses moyens sont vastes et qu'ils ont été en mesure de s'assujettir le monde pour en faire un miroir d'elle-même. Un cadre normatif ne tendant jamais que vers la confirmation de luimême et vers sa propre reproduction - comme du reste n'importe quelle entité organisée et vivante - il tendra aussi à s'identifier avec les moyens de son inscription.

De là, cette conséquence: l'assujettissement du monde par le décuplement des moyens au service de l'inscription signifie que le monde devient lui-même un moyen ${ }^{8}$. Tout devient médiation instrumentale au service d'une conquête qui, à travers l'altérité culbutée du monde, fonce toujours plus vite en direction d'ellemême. Le cadre normatif tend vers le solipsisme; et il le fera d'autant plus qu'il peut tout transformer en moyen de le faire. La langue devient un moyen de communication; le savoir, un moyen de produire; l'art, un moyen de se distraire en attendant de reprendre le travail d'instrumentalisation.

Les moyens ne se déployant jamais qu'en direction d'euxmêmes, la médiation instrumentale au service de l'inscription est

peut-être les prolégomènes d'une telle inscription. Sur le plan sociologique, la pratique du graffiti, assez répandue chez les jeunes de nos sociétés, serait une inscription dans le monde et une attestation identitaire alternative. Sur ce sujet, on peut consulter les ouvrages de David Le Breton, La chair à vif. Usages médicaux et mondains du corps humain, Paris, Métallié, 1993, et Corps et sociétés. Essai de sociologie et d'anthropologie du corps, Paris, Méridiens Klincksieck, 1985. De la même façon, la réflexion esthétique a reconnu depuis longtemps dans le barbeau jubilatoire des jeunes enfants la marque inaugurale du geste créateur et en a même reconnu la systématisation dans la pratique d'artistes comme Jackson Pollock.

8. Martin Heidegger, "La question de la technique", dans Essais et conférences, Paris, Gallimard, 1958, p. 9-48]; Jurgen Habermas, Connaissance et intérêt, Paris, Gallimard, coll. "Bibliothèque de philosophie", 1976; Gilbert Hottois, Le signe et la technique, Paris, Aubier, 1984. 
14

ainsi une pensée du Même ${ }^{9}$ qui ordonne toutes les différences en réseau et qui, ce faisant, les domestique.

\subsection{L'attestation de la vérité}

L'inscription d'un cadre normatif dans la réalité ne suffit pas à la fondation d'un ordre politique. L'inscription dans la réalité permet de confirmer à l'infini le cadre normatif; elle ne permet pas de l'établir. Il faut encore que ce cadre soit attesté à travers un événement. Pourquoi?

C'est que tout cadre normatif est impuissant à se fonder par ses propres moyens. L'ordre qu'il définit n'étant valide qu'à l'intérieur de ses propres limites, il ne saurait invoquer le régime de nécessités qu'il met en place pour justifier sa propre création. Quelle loi en effet pourrait sanctionner la création d'un nouvel ordre puisque toutes les lois qui pourraient le faire commencent avec lui?

De la même façon, en science, l'établissement d'une loi passe par un irréductible saut qualitatif, un pari interprétatif par lequel la nature même de l'énoncé concernant la réalité change de nature. Entre l'observation empirique et réitérée de tel phénomène et la généralisation de ce dernier dans une loi, il y a un passage à la limite ${ }^{10}$. Une série d'expériences réalisées dans les mêmes conditions a permis, par exemple, de constater, à de multiples reprises que l'eau se met à bouillir à $100{ }^{\circ} \mathrm{C}$. De là, on tire un énoncé à caractère général et normatif qui stipule que l'eau doit bouillir à cette température. Mais, à strictement parler, rien ne nous assure vraiment qu'il en sera de même à la prochaine occasion. Non pas que la généralisation inductive soit illégitime, mais il se passe quelque chose quand un énoncé dès l'abord inductif se met tout à coup à fonctionner sur le mode déductif. La possibilité d'énoncer la loi et d'incorporer un énoncé au règne du Même suppose l'exercice d'un certain arbitraire que l'on peut certes légitimer $a$ posteriori, mais dont la nature commence par être irréductible à l'ordre qu'elle fonde. Dans le pari interprétatif, on enjambe un

9. Vincent Descombes, Le même et l'autre. Quarante-cinq ans de philosophie française (1933-1978), Paris, Minuit, coll. "Critique", 1979.

10. Ludwig Wittgenstein, De la certitude [1969], Paris, Gallimard, coll. "Idées", 1976. 
vide, un espace non thématisable; on traverse une discontinuité et, celle-ci franchie, on se retourne pour en faire un point d'appui. Un point que rien ne distingue d'abord dans une dispersion aléatoire devient ainsi le premier d'une série ordonnée. Ce point est une limite et le pari interprétatif qui permet de poser une loi est lui-même passage par ce point-limite. Les règles de construction du représentable étant établies avec la fondation et s'appliquant de proche en proche, elles déploient nécessairement la transparence du Même. Dès lors que les prémisses de l'ordre sont posées, le reste est un emboitement de conséquences, une prolifération du nécessaire. Mais cet acte qui inaugure la juridiction du Même lui est dès l'abord étranger.

Le cadre normatif ne peut coloniser rétroactivement l'espace non balisé dont il est issu. Il vaut à partir du moment où il est posé, et à l'intérieur de la limite que dessine l'événement de la fondation. C'est donc dire que la nécessité n'est nécessaire que par le fait d'un événement qui est lui-même arbitraire et que le Même se déploie sur fond d'altérité. L'événement qui fonde est lui-même sans fondement. À lui vient s'adosser la nécessité interne du cadre normatif; mais l'événement, quant à lui, ne repose sur rien si ce n'est sur un acte qui s'autorise de lui-même. La fondation est un acte autothétique.

\section{3. Événement et fondation}

\subsection{Fondation et pacte intersubjectif}

Le cadre normatif doit être fondé mais il ne peut le faire par lui-même dans la mesure où l'événement de cette fondation lui est irréductiblement étranger. Comment alors établir la vérité des moyens par lesquels le cadre normatif pourra s'inscrire dans la réalité et produire à son tour du savoir?

Ce qu'il n'a pas, il faut le lui prêter, c'est-à-dire lui faire crédit d'une vérité dont il pourra par la suite nous rembourser. Or, ce crédit qui lui est accordé est toujours le fait d'un pacte intersubjectif dans lequel les individus, se prenant à témoin les uns les autres, attestent la vérité de ce qui commence.

La vérité ici en jeu n'est pas celle de l'adéquation empirique mais celle de la proclamation. La chose devient vraie d'avoir été proclamée; à charge, par la suite, à l'inscription dans la réalité de payer les dividendes qui sont dus. La vérité commence avec le 
fait d'être dite et reçue entre deux sujets - un "je" et un "tu" dont l'un devient le débiteur de l'autre, et devant l'instance impartiale d'un troisième sujet — un "il" — qui représente la communauté prise à témoin. Toujours dans l'établissement d'un ordre politique, il y a cette structure ternaire de l'attestation ${ }^{11}$.

Que l'un y soit le débiteur de l'autre ne dit rien d'autre que l'inachèvement de tout savoir, la finitude de l'individu qui ne possède pas sa propre origine, et son inscription dans une filiation qui doit être assumée pour elle-même. Sur le seul plan temporel, nous sommes tenus de nous en remettre à ceux qui sont venus avant nous, et de les croire. C'est le point de départ obligé sans lequel le doute, impuissant à se sortir de lui-même, se généralise et supprime toute possibilité de communication avec autrui. L'établissement de la vérité passe par l'assomption de notre finitude; et, réciproquement, la limite constitutive de tout savoir réclame l'établissement d'une vérité; laquelle ne saurait jamais être démontrée, mais sentie, consentie et qui, en tant que telle, pourrait se passer de tout contenu.

Dans le système des méridiens, il y a une convention qui établit le point 0 du cadre de référence à Greenwich en Angleterre. Sans cette convention, il n'y a pas d'orientation possible dans l'espace. La grille de méridiens n'étant pas amarrée à la surface du monde, une coordonnée désigne aussi bien un point qu'un autre et ne veut plus rien dire.

Or que dit au juste cette convention? Rien si ce n'est: "Ici tout commence". Le point 0 est un lieu vide et arbitraire. Mais en même temps, il ne tient sa fonction que du crédit qu'on lui accorde, c'està-dire de la force - en l'occurrence ici, les canonnières de la flotte britannique - du pacte intersubjectif qui l'établit. C'est à partir de lui que l'orientation et la mesure deviennent possible. Il en est de même pour l'ordre politique où norme, mesure, métrique, règle, loi commencent de la même façon par un pacte intersubjectif.

Seule cette fiducie inaugurale peut surmonter l'impossibilité du Même à accoucher de lui-même; seul le croire est en mesure d'adosser le savoir à une origine où se côtoient le Même et l'Autre. Un ordre politique ne peut commencer en effet qu'en venant d'un temps où il n'est plus et en allant au-devant d'un

11. Voir Dany-Robert Dufour, Les mystères de la trinité, Paris, Gallimard, 1990. 
temps où il n'est pas encore. Par l'événement d'une origine qui l'inscrit dans le temps et dans l'espace, le cadre normatif cesse d'être une fiction et devint un ordre politique; mais, ce faisant aussi, l'événementialité ouvre en lui une indépassable béance par laquelle l'Autre a prise sur lui et le dépossède de lui-même ${ }^{12}$. L'altérité ayant ainsi une part essentielle à la genèse du Même, le croire assume cette altérité dans l'arbitraire fondateur de tout savoir et s'en remet de ce qu'il sait à la communauté dont il tient ce savoir. Parce que le croire est le pli à partir duquel le savoir est rabattu sur lui-même comme un ourlet (le savoir est vrai parce que c'est le savoir) le croire assure la limite du cadre normatif et en assume la finitude. Sans ce pli, la trame du savoir irait en s'effilochant partout à sa frange. Incapable de surmonter le doute qui l'assaille continuellement à sa limite, partout où le savoir encore hypothétique n'est pas assuré, ce que l'on sait sombrerait à coup sûr dans ce que l'on ne sait pas sans la décision de surseoir à la complétude et de cheminer vaille que vaille avec ce qu'il faut bien appeler des "convictions". Le savoir ne peut être indubitable qu'à tout savoir, c'est-à-dire à être infini. Autrement, il lui faut assumer son événementialité et croire en lui-même.

\subsection{Pacte intersubjectif et communauté croyante}

C'est par l'attestation de la vérité que l'inscription dans la réalité devient possible. Or, si cette attestation procède d'un pacte intersubjectif, on pourrait penser que cette dernière est préalable à ce pacte; mais il n'en est rien. Le pacte autour d'un "il y a" fondateur procède de la communauté en même temps qu'il l'établit. Ce qui vient avant le pacte n'est pas encore un groupe; tout au moins, pas une communauté, au sens où les individus qui en font partie y sont arbitrairement rassemblés. Et à supposer même que les individus y soient fonctionnellement ordonnés les uns aux autres, comme ce serait le cas dans une termitière, il n'y a pas de groupe tant que les individus ne sont pas voués à une existence qui les dépasse en même temps qu'elle les subsume. Un ordre politique est instauré en prenant à témoin dans l'événement fondateur les subjectivités éparses qui, du coup, par l'acte de reconnaissance, viennent se totaliser autour de lui et se fondent ensemble dans une

12. Paul Ricour, Soi-même comme un autre, Paris, Seuil, coll. "L'ordre philosophique", 1990. 
18

origine commune. De la sorte le cadre normatif cesse d'être un dispositif spéculatif pour s'incarner dans la chair, faisant du groupe le corps même de la vérité. C'est dans la vérité de l'attestation qu'il y a agrégation des individus et formation du lien social. La vérité n'est vraie en effet que parce que le groupe l'incarne dans son existence; et le groupe, en retour, n'existe que parce que la vérité lui donne corps et lui tend l'icône où il se totalise. L'attestation de la vérité est en même temps la vérité de l'attestation: l'une et l'autre sont réversibles dans le corps où elles s'incarnent.

Par son effet performatif ${ }^{13}$, le témoignage de foi met donc en circulation une parole instauratrice de la vérité. Le témoignage ne fonctionnant pas sur le mode dénotatif, il constitue plutôt une effectuation de la vérité. Il incarne à mesure ce dont il parle; et, ce faisant, le rend vrai.

Le christianisme est né justement avec le témoignage des apôtres, témoignage à propos d'un événement aussi inassignable que celui de la résurrection. Et il continue de vivre par la parole que, selon le vœu de Jésus, l'on ne cesse de se répéter en mémoire de Lui. C'est le pacte intersubjectif des apôtres qui donne un statut fondateur à la proclamation de la résurrection. Et inversement, le corps du Christ ressuscité est ce par quoi un groupe de disciples, dès l'abord mis en déroute par l'échec de Jésus et dispersé par sa mise à mort, est de nouveau rassemblé et définitivement constitué en Église. L'immortalité du corps ressuscité devient pérennité de l'Église. Le Christ est vivant par son Église; et il n'y a d'Église que dans le Christ ressuscité.

Le même mécanisme est à l'œuvre dans tout corps politique. La personne du souverain dans les monarchies est l'incarnation du royaume: incarnation qui se réalise dans le faste protocolaire des innombrables cérémonies à la cour. De la même façon, aux ÉtatsUnis comme en France, l'image du président est investie du prestige de la nation entière. Ce qui a fait dire par certains analystes que la vie politique américaine était en vérité une religion civile ${ }^{14}$.

Quoique ne revendiquant pas toujours la même portée métaphysique - encore que, on parle parfois de la "France éternelle"

13. Pierre Bourdieu, Ce que parler veut dire, Paris, Fayard, 1984 ; John Langshaw Austin, Quand dire, c'est faire [1962], Paris, Seuil, coll. "L'ordre philosophique", 1970.

14. Peter Ludwig Berger (voir note 2). 
- la nation qui fait corps autour de symboles comme un drapeau, un hymne, un arc de triomphe ou l'autorité suprême du chef, n'est pas essentiellement différent de l'Église rassemblée autour du Christ et que la théologie de l'apôtre Paul désigne nommément comme le corps du Christ.

Nulle part sans doute plus clairement que dans le nationalsocialisme allemand avant et pendant la seconde guerre mondiale, le fondement symbolique du lien social a été à l'œuvre. Le nazisme a pris corps à travers de grandes liturgies où la scansion collective d'un certain nombre de slogans a pu ériger en vérité des contenus idéologiques dont la pauvreté ne laisse pas de nous frapper aujourd'hui encore. On ne peut comprendre autrement comment une population - sa classe intellectuelle y compris a pu, dans une aussi vaste proportion, emboîter le pas derrière un discours dont le contenu était de nature essentiellement phatique.

C'est par le fait d'avoir été scandés et proclamés au cours des grandes messes collectives où elles sont devenues le lien commun, le geist, l'esprit qui fait être en vérité que l'idéologie nazie a pu non seulement se donner une certaine vraisemblance mais accéder au statut de vérité, voire même devenir la statue resplendissante de la vérité. Au sens propre comme au figuré, il y a eu là transsubstantiation: la parole incandescente du Führer s'offrant pour être l'Incarnation de la Nation; et réciproquement, les foules déclamantes s'offrant à leur tour pour devenir l'Incarnation de sa Parole.

Un état en effet ne peut partir à la conquête du monde mû par la seule coercition qu'il est en mesure d'exercer sur l'ensemble du groupe. Il y faut beaucoup plus. Il y faut cette impulsion de tout le corps social que seul peut donner l'événement de la fondation quand on a le dispositif rituel pour s'y rapporter.

\section{Finitude et fondation}

\subsection{Le paradoxe de l'événement}

Du coup, l'événement de la fondation a un statut essentiellement paradoxal, participant à la fois de la nécessité qu'il inaugure et de l'arbitraire qui le fonde. Comme une limite, il est impossible en effet de dire si l'événement de la fondation est à l'intérieur ou à l'extérieur du cadre normatif. Pour autant qu'il est le premier 
jalon de celui-ci, il appartient au domaine du Même et en est un relais parmi d'autres; mais dans la mesure où cet événement est sans précession logique, son statut est absolument unique et appartient au domaine de l'Autre.

Le cadre normatif étant un dispositif classificatoire de l'expérience, sa vocation est générale. Pour qu'il soit opératoire, les grandeurs qu'il classe doivent être semblables, répétables et regroupables dans une série de catégories. Or, si les grandeurs qu'il catégorise sont des singularités, il cesse de pouvoir les classifier et devient le simple calque de leur dispersion.

De la même façon, si l'une de ces grandeurs est absolument singulière, elle constitue à elle seule une catégorie sui generis réfractaire à la classification. Elle est à l'intérieur du dispositif classificatoire, tout en n'y étant pas. Et la catégorie où elle entre en est une tout en ne l'étant pas, acquérant ce faisant un statut limitrophe par rapport à l'ensemble du dispositif.

De quelque façon que l'on essaie de le penser, l'événement de la fondation n'est pas l'ordre qu'il fonde. En logique formelle où l'univers opératoire est fermé, la négation ne renvoie jamais qu'au deuxième terme d'une alternative: le domaine a toujours pour corrélât un co-domaine. Avec cette conséquence que la négation permet de définir un contenu propositionnel avec autant de clarté que l'affirmation. La portée de la négation est limitée par la frontière de ce qu'en logique on appelle "l'univers". Nie-t-on cette négation que le contenu de la proposition rebrousse chemin vers son point de départ. Une double négation équivaut à une affirmation.

Alors que la situation ici est tout autre. La négation ouvre plutôt un espace non-thématisable de virtualités où elle sert de vecteur. La négation permet de problématiser le fait de la limite et ouvre le Même à sa différence. La négation ici ne se pose pas comme alternative. À proprement parler, elle n'a pas de contenu et ne peut donc fonctionner sur le mode dialectique. La négation de la négation ne fait guère de sens si ce n'est de réitérer l'injonction de l'événement. L'affirmation dit quelque chose; la négation ne le nie pas. Elle fait apparaître le dire. La négation ne modifie pas le dit, elle ouvre simplement la possibilité qu'il en soit autrement.

En un sens, la négation ne sort pas du Même. Longeant l'impossibilité d'en sortir, elle fait apparaître sa limite et contribue ainsi à son détournement. 
L'événement de la fondation est de la sorte la limite qui circonscrit le dispositif du cadre normatif et qui, en même temps, l'ouvre. Dedans/dehors, elle assure l'intégrité du Même et en même temps son commerce avec l'Autre. L'événement de la fondation est assise et béance ${ }^{15}$.

\subsection{Fondation et représentation}

L'opérativité des moyens par lesquels le cadre normatif peut s'inscrire dans la réalité et projeter sur elle le représentable commence avec l'événement de la fondation; mais l'opérativité finit aussi avec lui. Elle se reçoit de lui, mais bute aussi sur sa singularité.

Ce qui revient à dire que l'événement de la fondation est proprement irreprésentable. Non pas qu'il ne puisse pas être l'objet d'une catégorie dans le cadre normatif; mais catégorie sui generis - c'est-à-dire catégorie qui n'en est pas une - la représentation dont elle est l'objet se désavoue à mesure elle-même. La représentation est là non plus pour dire la réalité mais pour montrer la limite du représentable. Elle pose une borne silencieuse, aveugle et réfractaire à toutes les avances du cadre normatif ${ }^{16}$.

Ce qui ne veut pas dire que le cadre normatif ne puisse récupérer l'événement de fondation en y suspendant ces affichettes ou en dressant autour des tréteaux de marchands. (Rares en effet sont les cultures qui sont absolument iconoclastes. La plupart du temps, il faut pour retrouver l'âpreté de cette exigence, s'avancer jusque dans les contrées les plus lointaines de la mystique.) Mais l'événement de fondation est essentiellement dépris de ces représentations.

De deux choses l'une alors. Ou bien la représentation fonctionne, comme on vient de le dire, comme un dispositif déictique qui détourne de l'image et fait signe en direction d'un ailleurs radicalement étranger. (À cet égard, le registre des moyens rhétoriques à la disposition d'une telle auto-révocation correspond en gros à celui de la mythologie où la surenchère côtoie le paradoxe, où l'excès va de pair avec l'abstinence pour dire que l'événement de fondation n'est pas de ce monde.) Ou bien, la

15. Régis Debray, Critique de la raison politique ou L'inconscient religieux, Paris, Gallimard, 1987.

16. Le das chez Wittgenstein ne peut être "dit" mais seulement "montré" et appartient du coup au domaine de ce qu'il appelle "le mystique". 
représentation, au contraire, tente d'assurer l'origine et de la soustraire à l'événementialité en l'incorporant au Même. La représentation y passe au service de la métaphysique dont elle constitue une simple illustration. L'événement n'est convoqué que pour enfermer le cadre normatif en lui-même et désavouer toute altérité ${ }^{17}$. Mais dans ce dernier cas de figure, on peut faire confiance aux séismes de l'événementialité, aux revers imprévisibles de l'histoire pour lézarder l'habillage du cadre normatif et restituer l'arête vive de l'événementialité. Dès lors que l'histoire a des ratés qui bousculent les évidences de l'indubitable, c'est toujours la stèle de l'événement qui est remise à nu et nettoyer des bavardages du cadre normatif. Tant que l'ordre politique navigue sur la mer étale de la routine, les fonctionnaires ont beau jeu d'être les interprètes accrédités de la transcendance. Mais pour peu que la houle se lève, la parole redevient charismatique et passe aux prophètes, aux réformateurs et aux fondateurs. L'événement de la fondation redevient kairos.

\section{Sanctuarisation}

Du côté des choses, l'inscription réalise le cadre normatif; et du côté des corps, l'attestation l'incarne. Mais cette attestation adosse aussi le cadre normatif à son événementialité. L'attestation fonde mais, elle-même sans fondement, elle est aussi paradoxale et irreprésentable. En elle donc, le cadre normatif vient au-devant de son propre arbitraire. Le cadre normatif ne peut se fonder luimême et l'attestation qui lui fournit le sol où enfin se poser le lui dérobe en même temps.

En sorte que ce qui établit le cadre normatif est aussi ce par quoi il est transitoire et révocable. Pourquoi en effet ne pas remettre en question le pacte intersubjectif qui lie le groupe dès lors qu'il sert inégalement les intérêts de chacun? Pourquoi lui subordonner les intérêts individuels et accepter justement que le bien collectif prime sur le sien propre? Pourquoi le pacte aurait-il juridiction audelà de la génération qui l'a conclu pour engager l'avenir ${ }^{18}$ ?

17. Ce que Martin Heidegger appelle "l'oubli de l'être".

18. C'est précisément la question qui traverse l'œuvre entière d'Émile Durkheim. Dans Les formes élémentaires de la vie religieuse. Le système totémique en Australie [1915], Paris, PUF, 1960, ce qu'il appelle le "totem" est placé au centre du cadre normatif dont il constitue par ailleurs une représentation 
Pour surmonter la divergence centrifuge des intérêts, le pacte ne saurait survivre que s'il est mis à l'abri en dehors de l'espace social des marchandages et des tractations dans un lieu et un temps réservé où prévaut un registre de raisons plus hautes qui coupe court à toutes ratiocinations et referme la béance de l'événement. Or ce processus de confinement des valeurs fondatrices, nous l'appelons "sanctuarisation" et il constitue en propre la tâche du religieux avec son dispositif mythologique et rituel.

L'expression le dit bien: le sanctuaire est un espace à part et privilégié. Sacer d'où vient le mot "sacré" veut dire "séparé". Aussi, le sanctuaire est-il le gardien du sacré en dressant un périmètre d'exclusion entre lui et le reste du monde. Le sanctuaire limite.

En même temps, le sanctuaire désigne à la référence ce qui a une valeur éminente et exemplaire. Le sanctuaire indique une direction au regard en sorte que cette valeur, pour séparée qu'elle soit, reste malgré tout en rapport avec notre monde. Le sanctuaire montre.

S'il n'y avait que "séparation", la valeur serait perdue dans le lointain pour toute désignation; et s'il n'y avait au contraire que "désignation", la valeur disponible à tout venant serait absorbée par la proximité. Dans les deux cas, il n'y aurait plus de valeur. En somme, le sanctuaire est un dispositif qui tient le sujet à la bonne distance, ni trop loin, ni trop proche, au point exact de focalisation de l'effet de sens par lequel la valeur peut valoir.

Jusque là, le sanctuaire ne se distingue pas essentiellement du musée qui met en scène des valeurs esthétiques en montrant des ouvres et en dressant autour d'elle un périmètre d'exclusion qui les préserve. C'est du reste pourquoi la quincaillerie liturgique du sanctuaire peut elle-même devenir objet de représentation dans un musée; ou que l'on peut visiter la cathédrales de Chartres au même titre que le musée du Louvre.

Mais dans le sanctuaire, il ne s'agit pas d'isoler et de désigner au regard une valeur parmi d'autres. Le sanctuaire met en scène

magnifiée et transcendante. Là, dans cet "ailleurs", il survole les divergences d'intérêts et peut sacraliser le bien commun. 
et/ou produit par son dispositif la valeur des valeurs, celle qui soutient toutes les autres et qui rend possible leur évaluation. Il faut donc autre chose.

Dans le cas qui nous occupe, le pacte intersubjectif qui institue la nécessité doit devenir lui-même nécessaire, c'est-à-dire valoir au-delà de toutes autres valeurs. Le sanctuaire doit transformer le pacte intersubjectif en valeur-limite, terme premier d'une échelle sur laquelle les intérêts des uns et des autres viendront après. Par la sanctuarisation, valeur et limite doivent se confondre dans la fondation.

Le sanctuaire procédera donc en faisant jouer l'une sur l'autre limitation et monstration. Le sanctuaire montre la limite et il limite ce qu'il montre. En sorte que, de la valeur, il ne reste plus de contenu à représenter; de la valeur il ne reste plus que la valence, c'est-à-dire ce qui fait bord. Il ne reste que l'incandescence de l'événement de fondation.

En définitive, le sanctuaire désigne une borne silencieuse au pied de laquelle viennent mourir toutes les paroles; et en même temps, il opère le confinement dont cette borne est l'objet. En la soustrayant au regard, le sanctuaire transforme la borne en un espace vague et vide où toute parole se tient en réserve: sorte de sheol où, avant la séparation des eaux, plane encore l'esprit de Dieu, où toute parole coïncide avec le silence dans le Verbe.

Disons-les choses autrement. On a vu que l'événement de fondation adosse le cadre normatif à un espace non-thématisable de virtualités. Or, la sanctuarisation opère en redoublant cet espace de virtualités d'une boucle plus lointaine qui vient le circonscrire. Par la sanctuarisation, la limite devient simultanément l'englobé qui court entre les domaines de l'Autre et du Même, et l'englobant de toute altérité. La sanctuarisation fait opérer le paradoxe de la limite sur lui-même. L'ouvert/fermé de la limite devient le fermé/ouvert.

Du coup, l'événement de fondation, arbitrairement posé quelque part dans un espace de virtualités - et donc englobé ultime —, devient l'horizon de toutes possibilités, c'est-à-dire l'englobant ultime. Toute possibilité est épuisée à l'avance dans l'événement de fondation. Par la sanctuarisation, l'arbitraire coïncide avec le nécessaire. 
Concrètement, dans la symbolique médiévale ${ }^{19}$, la cathédrale est considérée comme un centre du monde. Point de référence dans l'étendue, la cathédrale permet d'organiser l'espace. Elle introduit dans l'homogénéité initiale de l'espace un point de référence absolu par rapport auquel l'orientation devient possible. On dit alors de la cathédrale qu'elle est un axis mundi ${ }^{20}$.

Mais le même symbolisme affirme concurremment que la cathédrale est aussi une imago mundi, c'est-à-dire une représentation miniature de l'univers, un microcosme. Ce qui veut dire que l'univers entier est contenu en elle, et que l'enceinte de la cathédrale se confond avec les confins ultimes de l'univers. La cathédrale est en même temps le premier englobé et le dernier englobant et sature d'un seul coup la totalité de l'espace avec sa présence. Le sacré est partout.

Toute possibilité est comprise à l'avance dans le paradoxe de sa présence. Le redoublement de l'espace de virtualités autour de la cathédrale par la circonférence du Même, capture l'événementialité et l'englobe à l'avance dans l'événement de fondation. La fondation devient la forme achevée dans laquelle s'épuise toute événementialité.

La sanctuarisation est bien entendu une machinerie spatiale, comme on vient de le voir avec la cathédrale; mais elle est aussi à l'œuvre partout où il s'agit d'assurer les conditions de possibilité du cadre normatif. Aussi, soutient-elle le fonctionnement du mythe et du rituel.

À l'intérieur d'une culture le mythe ${ }^{21}$ a en effet pour fonction de raconter les origines du monde; non pas seulement du

19. Mary-Madeleine Davy, Initiation à la symbolique romane, Paris, Flammarion, 1967; Cosmas Indicopleustès, Topographie chrétienne, Paris, Cerf, 1968; Marc de Smedt, Le lieu du temple. Géographie sacrée et initiation, Paris, Albin Michel, coll. "Question de", 1988.

20. Mircea Eliade, "Architecture sacrée et symbolisme", dans Mircea Eliade, Paris, l'Herne, 1978, p. 141-153; Mircea Eliade, Le sacré et le profane, Paris, Gallimard, coll. "Idées", 1965; Mircea Eliade, Le chamanisme et les techniques archaïques de l'extase, Paris, Payot, 1951; Mircea Eliade, Méphistophélès et l'androgyne, Paris, Gallimard, coll. "Idées", 1962.

21. Mircea Eliade, Aspects du mythe, Paris, Gallimard, coll. "Idées ", 1963; Georges Gusdorf, Mythe et métaphysique. Introduction à la philosophie, Paris, Flammarion, 1953. 
cosmos dans son ensemble mais aussi de toutes et chacune des réalités signifiantes qui le composent. Il y a un mythe pour l'origine de la mort; un autre pour l'origine de la sexualité, de la chasse, et ainsi de suite. Toutes les réalités actuelles y sont répertoriées et mises en ordre. Le mythe est ainsi un inventaire des représentations de notre monde et, en tant que tel, le récit étiologique du cadre normatif.

Or, comme le sanctuaire, le mythe limite et montre. Le mythe limite en ceci qu'il introduit une différence radicale entre les événements d'alors et ceux d'aujourd'hui. Il met en scène des personnages hors du commun qui ont disparu une fois pour toutes de notre monde. On pourra bien les réactualiser par le rituel; mais, ce faisant, on n'aura jamais fait que rendre présent l'exemplaire unique de l'origine. Par le mythe, l'événement de fondation est mis à l'abri dans une temporalité lointaine et révolue. Le mythe institue une limite.

Par ailleurs, le mythe raconte bien un certain nombre de choses; mais, ce faisant, il ne fait que montrer. Cette fonction de dire, dans la mesure où il la partage avec d'autres formes de récit, il ne la possède pas en propre. Ce que le mythe a de spécifique, c'est de montrer l'événement de fondation. Au fond, en nous disant que les choses sont comme cela parce qu'elles sont comme cela, le mythe n'ajoute rien au monde. Il ne fait qu'en marteler la contingence et la sceller dans la forme définitive de l'origine. Le monde est certes le fait d'un coup de dés mais le hasard y est à tout jamais épuisé parce que c'est la transcendance qui l'a lancé.

De la même façon, le rituel ${ }^{22}$ est à la fois un dispositif déictique et de confinement. Le luxe surérogatoire, la surenchère gratuite de la mise en scène rituelle est simplement faite pour montrer que: "Ici, il y a quelque chose". Toujours en excès par rapport à l'utilité fonctionnelle du geste, le rituel démarque en même temps un espace réservé, isolé du quotidien. Drapé dans la pompe du rituel, le pacte intersubjectif est simplement mis à l'abri et désigné à la référence. Et quand le rituel en vient à montrer la limite, le pacte devient absolu.

22. Jean Cazeneuve, Sociologie du rite. Tabou, magie, sacré, Paris, PUF, 1971. 


\section{Conclusion}

La tâche en propre du religieux étant de gérer ce que l'événement de fondation a de proprement paradoxal et d'irreprésentable, on aura compris que le politique ne peut se fonder sans générer une mythologie et des rituels.

Que dans une culture, telle religion assume la tâche fondationnelle du politique ne veut pas dire qu'elle doive le faire partout et qu'il ne puisse y avoir d'ordre politique sans religion. Ce que nous voulons dire c'est que les formes constitutives du religieux peuvent se retrouver ailleurs et se déplacer dès lors que la religion instituée manque à sa fonction ou qu'un ordre politique quelconque choisit de se séculariser. On verra spontanément apparaitre alors un processus de sanctuarisation par lequel l'ordre politique mettra à l'abri le pacte intersubjectif qui le fonde et le désignera à l'exemplarité, par lequel aussi la vérité de ce pacte s'incarnera dans le corps social.

Nos démocraties ont en effet leur propre mythologie; elles ont aussi la liturgie par laquelle elles peuvent surmonter la béance de leur commencement, faire surgir l'angoisse qui les travaille pour l'insuffler dans l'icône d'un corps glorieux où elle trouve un abri définitif et auquel, en retour, elle prête une vie qui n'est pas la sienne. 